\title{
ELMO2 association with Gai2 regulates pancreatic cancer cell chemotaxis and metastasis
}

\author{
Yecheng Wang ${ }^{1}$, Hongyan $\mathrm{Li}^{1}$, Fei Li ${ }^{\text {Corresp. } 1}$ \\ ${ }^{1}$ Department of General Surgery, Xuanwu Hospital Capital Medical University, Bei Jing, China \\ Corresponding Author: Fei Li \\ Email address: xw_lifei36@163.com
}

Background. Pancreatic cancer is a highly lethal disease. Nearly half of the patients have distant metastasis and remain asymptomatic. Emerging evidence suggests that the chemokine, CXCL12, has a role in cancer metastasis. The interaction between CXCL12 and CXCR4 activates heterotrimeric G proteins, which regulates actin polymerization and cancer cell migration. However, the molecular mechanisms underlying pancreatic cancer cell migration are still largely obscure. Here, we addressed the role of ELMO2 in chemotaxis and metastasis of pancreatic cancer cells.

Methods. Pancreatic cancer cell lines PANC-1 and AsPC-1 and siRNA-mediated knockdown of ELMO2 were used to determine the effects of ELMO2 on cancer cell chemotaxis, invasion, migration. Coimmunoprecipitation assays were carried out to identify interacting partners of ELMO2.

Results. ELMO2 knockdown inhibited pancreatic cancer cell chemotaxis, migration, invasion, and F-actin polymerization. Co-immunoprecipitation assays revealed that ELMO2 interacted with Gai2 and that CXCL12 triggered Gai2-dependent membrane translocation of ELMO2. Thus, ELMO2 is a potential therapeutic target for pancreatic cancer. 


\title{
1 ELMO2 Association with Gai2 Regulates Pancreatic Cancer
}

2 Cell Chemotaxis and Metastasis

3

4 Yecheng Wang ${ }^{1}$, Hongyan $\mathrm{Li}^{1}$, $\mathrm{Fei} \mathrm{Li}^{1}$

$5 \quad{ }^{1}$ Department of General Surgery, Xuanwu Hospital, Capital Medical University, Beijing, China

6 Corresponding author:

7 Fei Li

8 Xuanwu Hospital, Capital Medical University, Beijing 100053, China

9 Email: xw_lifei36@163.com
11

\begin{abstract}
Background. Pancreatic cancer is a highly lethal disease. Nearly half of the patients have distant metastasis and remain asymptomatic. Emerging evidence suggests that the chemokine, CXCL12, has a role in cancer metastasis. The interaction between CXCL12 and CXCR4 activates heterotrimeric $\mathrm{G}$ proteins, which regulates actin polymerization and cancer cell migration. However, the molecular mechanisms underlying pancreatic cancer cell migration are still largely obscure. Here, we addressed the role of ELMO2 in chemotaxis and metastasis of pancreatic cancer cells.

Methods. Pancreatic cancer cell lines PANC-1 and AsPC-1 and siRNA-mediated knockdown of ELMO2 were used to determine the effects of ELMO2 on cancer cell chemotaxis, invasion, migration. Co-immunoprecipitation assays were carried out to identify interacting partners of ELMO2.

Results. ELMO2 knockdown inhibited pancreatic cancer cell chemotaxis, migration, invasion, and F-actin polymerization. Co-immunoprecipitation assays revealed that ELMO2 interacted with Gai2 and that CXCL12 triggered Gai2-dependent membrane translocation of ELMO2. Thus, ELMO2 is a potential therapeutic target for pancreatic cancer.

Keywords: Pancreatic cancer, ELMO2, Gai2, Metastasis, Cell migration, Chemotaxis

\section{Introduction}

Pancreatic cancer is one of the most malignant cancers of the digestive system. Currently, it is the fourth leading cause of cancer-related death due to early invasion and rapid metastasis (Kleeff et al. 2016; Siegel et al. 2018). Because of the lack of reliable markers for early diagnosis and aggressive tumor biology, the 5-year overall survival rate is still extremely low, and, despite important clinical advancements, the outcome is unfavorable in most patients (Conroy et al. 2016; Garrido-Laguna \& Hidalgo 2015). Therefore, it is of vital importance to discover and characterize the molecular mechanisms underlying pancreatic cancer cell migration and
\end{abstract}


metastasis. This may help identify novel factors involved in multi-step tumor metastasis and improve the treatments.

ELMO (Engulfment and Cell Motility) is a family of related scaffold proteins involved in intracellular signaling networks and with a high degree of evolutionary conservation. In mammals, the ELMO protein family consists of three isoforms: ELMO1, ELMO2, and ELMO3. As the mammalian homologs of Caenorhabditis elegans CED-12, the ELMO proteins play a major role in cell migration and cytoskeletal rearrangements (Gumienny et al. 2001). Although they lack intrinsic catalytic activity, ELMO proteins can function as adaptors to regulate the activity of plasma membrane and cytoplasmic proteins (Patel et al. 2011). Previous studies have shown that ELMO protein interactions with a number of different proteins activate signaling pathways that cause cell migration or promote cell movement. Proteins interacting with ELMO, such as Gai2, G $\beta \gamma$, and Nck-1, are mostly cell membrane-associated and involved in the regulation of cytoskeletal organization (Fritsch et al. 2013; Li et al. 2013; Zhang et al. 2014). Interestingly, ELMO family members such as ELMO1 and ELMO3 have been implicated in a variety of malignant cancers, such as glioma, breast cancer, colorectal cancer, hepatocellular carcinoma, and non-small-cell lung carcinoma (Fan et al. 2015; Jarzynka et al. 2007; Jiang et al. 2011; Peng et al. 2016; Zhang et al. 2015). In previous studies, the researches mostly focused on the function of ELMO1 and EMLO3 in tumor development, invasion, and formation of metastasis. However, the studies about the role and function of ELMO2 were very few. Early reports only showed that ELMO2 localized to cell-cell contacts regulating both integrin- and cadherin-based adhesions, which facilitated to reposition the cells from migration to strong cellcell adhesion (Toret et al. 2014). Unfortunately, the biological behaviors and molecular mechanisms of ELMO2 remained unclear. To our knowledge no previous studies have illustrated a relationship between ELMO2 and invasiveness in pancreatic cancer. This study aimed to fully understand the function and mechanisms of ELMO2 in pancreatic cancer chemotaxis and metastasis.

G proteins, also known as guanine nucleotide-binding proteins, are a family of molecular transducers involved in the transmission of signals generated by a variety of stimuli, such as chemokines, neurotransmitters, and hormones. G proteins are typically represented by the membrane-associated heterotrimeric $\mathrm{G}$ proteins, which are activated by $\mathrm{G}$ protein-coupled receptors (GPCRs), and are engaged in cell signaling. Heterotrimeric $G$ proteins consist of three major subunits, alpha $(\alpha)$, beta $(\beta)$, and gamma $(\gamma)$. During the past two decades, the function of $G$ proteins has been extensively investigated. Signaling molecules like chemokines bind to the extracellular GPCR domain, after which an intracellular domain facilitates the dissociation of the heterotrimeric G proteins, Gai and $\mathrm{G} \beta \gamma$, which in turn activates a cascade of intracellular signaling events (Fraser 2008; Xu et al. 2010). GPCRs and G proteins may cooperate in the regulation of cell actin cytoskeleton. The accumulation of actin filaments at leading-edge protrusions of the cell membrane increases cell mobility and promotes cell migration (Hurst \& Hooks 2009; Kim et al. 2013; Muller et al. 2001). However, little information is available on the role of $\mathrm{G}$ proteins in the migration and metastasis of pancreatic cancer cells.

In metastasis, cancer cells detach from the primary tumor, travel through the bloodstream or 
lymph system, and form a new tumor in other organs or tissues. To metastasize or spread, cancer cells need to invade, escape from a proper vessel, and settle at a distant site (Condeelis \& Segall 2003). Chemotaxis is the movement of an organism or cell in response to a chemical stimulus (Iglesias \& Devreotes 2008). Chemokines are a family of small cytokines, signaling proteins secreted by a variety of cells. They induce chemotaxis by interacting with specific chemokine receptors on the surfaces of target cells. The crucial role of chemotaxis in the recruitment of inflammatory cells to infection sites is a long-established concept (Jin et al. 2009). Interestingly, recent studies have shown that chemotaxis is critical for cancer cell dissemination (Condeelis et al. 2005; Murphy 2001). A complex network of chemokines contributes to chemotaxis in tumor cells, regulating cancer cell growth, invasion, and metastatic progression (Balkwill 2004; Swaney et al. 2010). A particularly important role in chemotaxis is played by the chemokine receptor, CXCR4, and by its ligand, CXCL12 (also known as SDF1, stromal cell-derived factor 1), which initiate directed cell migration in various kinds of cancer (Archibald et al. 2012; Hu et al. 2014; Li et al. 2014; Yang et al. 2015). The CXCR4/CXCL12 interaction triggers downstream signaling cascades that may promote metastatic progression (Guyon 2014). However, the molecular mechanism by which this complex affects metastasis in pancreatic cancer remains to be elucidated.

In this study, we investigated the role of ELMO2 and CXCL12 in pancreatic cancer using cancer cell lines. The results of this study are expected to provide novel insights into the metastatic progression of pancreatic cancer cells.

\section{Materials \& Methods}

\section{Cell Culture}

The pancreatic cancer cell lines, PANC-1 and AsPC-1, were purchased from American Type Culture Collection (Manassas, VA, USA). All pancreatic cancer cell lines were cultured in RPMI-1640 medium (Hyclone, Shanghai, China) supplemented with 10\% fetal bovine serum (FBS; Gibco Invitrogen Corporation, Australia) and were incubated at $37{ }^{\circ} \mathrm{C}$ in a humidified atmosphere containing $5 \% \mathrm{CO}_{2}$.

\section{Western blotting}

After indicated treatments, cells were collected and total proteins were extracted with PMSF (Beyotime Biotechnology, shanghai, China) containing RIPA lysis buffer (Beyotime Biotechnology, shanghai, China) as instructed. Protein samples were quantified by Pierce ${ }^{\mathrm{TM}}$ BCA Protein Assay Kit (Thermo Scientific) via spectrophotometer (Thermo Scientific, USA) at $562 \mathrm{~nm}$. Protein loading buffer (Sigma) was applied to denature protein samples. Total protein samples $(20 \mu \mathrm{g} /$ lane $)$ were separated by $10 \%$ sodium dodecyl sulfate polyacrylamide gel electrophoresis (SDS-PAGE), and transferred to a polyvinylidene difluoride membrane (GE Healthcare Amersham ${ }^{\mathrm{TM}}$ Hybond $^{\mathrm{TM}}$ ). The membranes were blocked with 5\% skim milk for one hour at room temperature and incubated with the primary antibodies anti-ELMO2 (ab2240, 
Abcam), anti-Gai2 (sc-13534, Santa Cruz Biotechnology) or anti-GAPDH (ab8245, Abcam) at 4 ${ }^{\circ} \mathrm{C}$ overnight, and then were washed three times with PBS containing $0.1 \%$ Tween-20 (PBST). The membranes were then incubated with the horseradish peroxidase-conjugated secondary antibodies anti-goat (ab6885, Abcam), anti-mouse (sc-516102, Santa Cruz Biotechnology) or anti-rabbit (ab6721, Abcam) for 1hour at room temperature. Finally, the bound proteins were visualized using the SuperSignal ${ }^{\mathrm{TM}}$ West Pico PLUS Chemiluminescent Substrate (Thermo Scientific) and analyzed with FluorChemHD2 system (ProteinSimple, USA).

\section{Transient Transfection}

Pancreatic cancer cell lines were cultured until they reached $60-80 \%$ of confluence before transfection. To reduce the expression of ELMO2 and Gai2, specific siRNAs were used for in vitro transfection. Cells were then incubated for $48 \mathrm{~h}$, followed by protein expression analysis by western blotting. The sequences of ELMO2 siRNA were 5'CCCAGAGUAUUAUACCCUCCGUUAU-3', 5'-CCCACUACAGUGAGAUGCUGGCAUU3', and 5'-CACAUCAAUCCAGCCAUGGACUUUA-3'. The sequences of Gai2 siRNA were 5'GAGGACCUGAAUAAGCGCAAAGACA-3', 5'-ACGCCGUCACCGAUGUCAUCAA-3', and 5'-CCGACACCAAGAACGUGCAGUUCGU-3'. To enhance the expression of ELMO2 and Gai2, the overexpression plasmids GV362 and GV141, respectively, were transfected into PANC-1 cells. Both plasmids were purchased from Genechem Co., Ltd (Shanghai, China). All transfections were performed using Lipofectamine 3000 reagent (Invitrogen) in accordance with the manufacturer's instructions. For the knockdown of ELMO2 throughout the studies, we chose siRNA_1 whose sequence was 5'-CCCAGAGUAUUAUACCCUCCGUUAU-3' to perform the following experiments.

\section{Exogenous Co-immunoprecipitation (Co-IP)}

PANC-1 cells were plated in 10-cm culture dishes before transfection. To obtain a high level of exogenous ELMO2 and Gai2 expression, PANC-1 cells were transfected with GV362 FlagELMO2 and GV141 Flag-Gai2, respectively. Cells were collected and total proteins were extracted with PMSF (Beyotime Biotechnology, shanghai, China) containing RIPA lysis buffer (Beyotime Biotechnology, shanghai, China). First, we incubated the cell lysates with monoclonal anti-flag antibodies (cat. F3165, Sigma) with continuous mixing overnight. Cell lysates were also immunoprecipitated with control rabbit IgG antibodies (CST-2729, Cell Signaling Technology). Next, PureProteomeTM protein A/G mix magnetic beads (Merck-Millipore) were added to the antibody-antigen complex and subjected to continuous mixing. Third, the precipitates were eluted from the magnetic beads by boiling in electrophoresis sample buffer, separated by SDSPAGE, and detected with anti-Gai2 (sc-13534, Santa Cruz Biotechnology) and anti-ELMO2 (ab2240, Abcam) antibodies.

\section{Immunofluorescence}

Briefly, PANC-1 cells were plated in 24-well plates containing round glass coverslips (one per well) and incubated for $24 \mathrm{~h}$ to obtain stable attachment to the glass coverslips. Before 
160

161

162

163

164

165

166

167

168

169

170

171

172

173

174

175

176

177

178

179

180

181

182

183

184

185

186

187

188

189

190

191

192

193

194

195

196

197

198

199

200

stimulation with CXCL12 (R\&D Systems, Inc.), cells were serum-starved for $3 \mathrm{~h}$, followed by incubation with CXCL12 (100 ng/ml) at $37^{\circ} \mathrm{C}$ for $1 \mathrm{~h}$. A solution containing $4 \%$ paraformaldehyde was used for cell fixation. Cell membranes were permeabilized with $0.1 \%$ Triton X-100. Donkey serum was used for blocking non-specific interactions, based on the species in which the secondary antibody was raised. After the blocking step, cells were incubated with diluted primary antibodies anti-ELMO2 (ab2240, Abcam) or anti-Gai2 (sc-13534, Santa Cruz Biotechnology) overnight.

The cells were subsequently stained with Alexa Fluor 488-conjugated secondary antibody (cat. A32814, Invitrogen) or 546-conjugated secondary antibody (cat. A10036, Invitrogen) for $1 \mathrm{~h}$ at room temperature. Finally, cells on coverslips were mounted and visualized using a Leica TCS SP5 II confocal microscope (Leica Microsystems CMS GmbH).

\section{Wound-Healing Assay}

Pancreatic cancer cell lines were seeded in 6-well plates and divided into three groups: normal, control, and siELMO2. Cells were cultured until they reached an 80-90\% density (ca. $24 \mathrm{~h}$ ). The cell monolayer was gently and slowly scratched with a $10-\mu 1$ pipette tip across the well. Then, the wells were gently washed twice with PBS to remove the detached cells. The medium was replaced by RPMI 1640 medium with 1\% FBS. Finally, photographs of the monolayer were taken with a microscope at various time points $(0,3,6,9,12$, and $24 \mathrm{~h})$.

\section{Chemotaxis Assay}

The Chemotaxis assays were performed as described by the manufacturer (Neuro Probe) and by Sun et al. (Sun et al. 2005). In this assay, a 48-well microchemotaxis chamber (AP48 chemotaxis chamber, Neuro Probe) was used, and prepared $50 \mu$ cell suspension $\left(2 \times 10^{5}\right.$ cells per ml $)$ were placed on the upper chamber and were allowed to migrate through the permeable filter membrane (PFB8, Polycarbonate membranes, 25x80mm, Neuro Probe) into the lower chamber. A solution containing the chemokine $(0,10,100,1,000 \mathrm{ng} / \mathrm{ml}$ CXCL12) was placed below the cell-permeable membrane. After a 3 -h incubation in $5 \% \mathrm{CO} 2$ at $37^{\circ} \mathrm{C}$, the cells that had migrated through the $8-\mu \mathrm{m}$ filter membrane were fixed, stained, and counted in three separate fields $(\times 400)$ by a microscope.

\section{Cell Invasion Assay}

Invasion assays were performed using a transwell chamber inserted with a polycarbonic membrane (cat. 3422 Corning, USA). To reproduce appropriate in vivo environments for 2D and 3D cell movements, we added $80 \mu \mathrm{l}$ of extracellular matrix (Corning 356234) into the upper compartment of the transwell cell culture inserts. CXCL12 $(0,10,100,1,000 \mathrm{ng} / \mathrm{ml})$ was added to the lower well of the plates as an attractant. $2 \times 10^{4}$ cells suspended in $100 \mu 1$ serum-free medium were seeded into the upper chamber. The plates were incubated for $24 \mathrm{~h}$ at $37^{\circ} \mathrm{C}$. Then, the cells on the lower side of the insert membrane were fixed. Finally, the cells on the lower side of the filter were counted under a microscope. 
201

202

203

204

205

206

207

208

209

210

211

212

213

214

215

216

217

218

219

220

221

222

223

224

225

226

227

228

229

230

231

232

233

234

235

236

237

238

239

240

\section{Adhesion Assay}

Briefly, a fibronectin (Sigma-Aldrich Corporation) solution was previously prepared and stored at $4{ }^{\circ} \mathrm{C}$. Then, 96-well plates (Costar-3599, Corning, US) were coated with fibronectin $(10 \mu \mathrm{g} / \mathrm{ml}$ in PBS) at room temperature for $1 \mathrm{~h}$. After coating, the fibronectin solution was removed. Thermally denatured BSA was added to the plates, followed by an incubation of $1 \mathrm{~h}$ at $37^{\circ} \mathrm{C}$. Then, the plates were washed twice with serum-free RPMI 1640 medium. CXCL12 (0, 10, 100, and 1,000 ng/ml) was added to the wells of fibronectin-coated plates. After the addition of the prepared $100 \mu \mathrm{l}$ cell suspension $\left(5 \times 10^{5}\right.$ cells per $\left.\mathrm{ml}\right)$, the plates were incubated at $37^{\circ} \mathrm{C}$ for $30 \mathrm{~min}$ and then washed thrice with PBS to remove non-adherent cells. Next, a $10 \mu \mathrm{l}$ Cell Counting Kit-8 (CCK-8) solution (Dojindo, Japan) was added to each well and incubated for $1.5 \mathrm{~h}$. Finally, the adherent cells were stained and quantified at OD450 using a Microplate Reader (Thermo) according to the manufacturer's instructions. The cell adhesion ratio of the assay was calculated according to the following formula: cell adhesion $(\%)=$ OD of the adhered cells $/ \mathrm{OD}$ of the total cells $\times 100 \%$.

\section{F-actin Polymerization Assay}

Cellular F-actin measurement was done as described by Tsuboi (Tsuboi 2006). Pancreatic cancer cells were stimulated with $100 \mathrm{ng} / \mathrm{ml} \mathrm{CXCL} 12$ for the designated time points at $37^{\circ} \mathrm{C}$. Then, the cells were fixed with $4 \%$ paraformaldehyde, permeabilized with $0.1 \%$ Triton X-100, and stained with Alexa Fluor 568-phalloidin (Invitrogen) for $60 \mathrm{~min}$ at room temperature. The level of Factin was measured by a microplate fluorescence reader. The results were expressed as relative F-actin values, as follows:

$\mathrm{F}$-actin $\mathrm{t} / \mathrm{F}$-actin $0=($ fluorescence $\mathrm{t} / \mathrm{mg} \mathrm{ml}-1) /($ fluorescence $\mathrm{t} 0 / \mathrm{mg} \mathrm{ml}-1)$.

\section{Statistical Analysis}

The statistical analyses were performed with GraphPad Prism 8 (La Jolla, CA, USA). The experimental data are expressed as the mean \pm SD. We designed each experiment with 3 replicates, which were done all at the same time. And each experiment was performed three times. One-way and two-way ANOVA were used to analyze the data. A p value below 0.05 was considered statistically significant.

\section{Results}

\section{Role of ELMO2 in the Migration and Chemotaxis of Pancreatic Cancer Cells}

To explore the role played by ELMO2 in the process of cell migration, we initially investigated its expression level in pancreatic cancer cell lines. The reasons why PANC-1 and AsPC-1 were chosen in this study were as follows: Firstly, information concerning the clinical course of the sites where cell lines were deprived from was important in defining the biologic and pathologic characteristics of the tumor cell lines. Both these two cell lines were derived from patients with an adenocarcinoma in the head of the pancreas and they shared similar phenotypic characteristics, such as adhesion, invasion and migration. Secondly, the cell population doubling 
241 times for PANC-1 and AsPC-1 were very close which made it more convenient for our

242 experimental operation. Small interfering RNA (siRNA) was used to suppress ELMO2

243 expression (Figure 1A). Then, a wound-healing assay was utilized to evaluate cell migration. The

244 decreased expression of ELMO2 reduced the migration capacity of PANC-1 and AsPC-1 cells

245 (Figure 1C). Moreover, a chemotaxis assay indicated that CXCL12 could distinctly enhance the

246 chemotactic ability of PANC-1 and AsPC-1 cells, while ELMO2 silencing inhibited the

247 CXCL12-induced chemotaxis in these cell lines (Figure 1B).

248

249

250

251

252

253

254

255

256

257

258

259

260

261

262

263

264

265

266

267

268

269

270

271

272

273

274

275

276

277

278

279

280

281

\section{Knockdown of ELMO2 Inhibited Invasion, Adhesion, and F-actin Polymerization in PANC-1 and AsPC-1 Cells}

Next, a cell invasion assay was performed to monitor the movement of pancreatic cancer cells through an extracellular matrix. We found that ELMO2 knockdown suppressed CXCL12induced invasiveness in both PANC-1 and AsPC-1 cells (Figure 2A). Furthermore, cell adhesion assay suggested that ELMO2 downregulation by siRNA weakened the adhesion ability of PANC-1 and AsPC-1 cells (Figure 2B). When CXCL12 combines with its receptor CXCR4, intracellular signaling events induce membrane protrusions because of actin polymerization. These events enhance the motility of cancer cells and promote chemotaxis and invasion. CXCL12 generated a transient F-actin accumulation in PANC-1 and AsPC-1 cells, in line with previous findings (Yan et al. 2012). Notably, F-actin filaments were clearly reduced in siELMO2 cells within $30 \mathrm{~s}$ (Figure 2C). These results suggested that ELMO2 knockdown inhibited F-actin polymerization in pancreatic cancer cells. Thus, ELMO2 might participate in CXCL12-mediated invasion.

\section{ELMO2 Interacts with Gai2}

Previous reports have shown that the association between ELMO1 and Gai2 contributes to actin polymerization in human breast cancer cells. Thus, it was reasonable to expect that Gai2 interacts with ELMO2 in pancreatic cancer cells. To verify this possibility, a Co-IP assay was performed. First, exogenous overexpression of ELMO2 was successfully induced by transfecting PANC-1 cells with the GV362-ELMO2-Flag plasmid. Then, ELMO2-Flag, along with its endogenous interactors, was captured from PANC-1 cell lysates using specific anti-Flag antibody. Interestingly, Gai2 was found among the ELMO2-interacting partners, as assessed by immunoblotting with Gai2 antibody (Figure 3A). Moreover, when Gai2-Flag was overexpressed following cell transfection with the GV141-GNAI2-Flag plasmid, endogenous ELMO2 was coprecipitated by the anti-Flag antibody along with Gai2-Flag (Figure 3B). Taken together, our results confirmed the physical association between ELMO2 and Gai2 in pancreatic cancer cells.

\section{Cell Stimulation with CXCL12 Results in ELMO2 Membrane Translocation}

To further investigate the interaction networks involving ELMO2 and Gai2, immunofluorescence microscopy was used to examine the subcellular localization of the two proteins. In unstimulated cells, clear Gai2-specific fluorescence, but not ELMO2 fluorescence, was detected at the plasma membrane. Interestingly, after CXCL12 stimulation, ELMO2 was 
282 also detected on the plasma membrane. According to the colocalization analysis performed on a

283

284

285

286

287

288

289

290

291

292

293

294

295

296

297

298

299

300

301

302

303

304

305

306

307

308

309

310

311

312

313

314

315

316

317

318

319

320

321

pixel by pixel basis, Gai2 and ELMO2 were clearly found to co-localize on the plasma

membrane after CXCL12 stimulation of pancreatic cancer cells (Figure 4A). Next, the impact of Gai2 silencing on ELMO2 localization, and vice versa was explored in PANC-1 cells transfected with the appropriate siRNAs (Figure 4B,C). Interestingly, the membrane translocation of ELMO2 was reduced in Gai2-knockdown cells, even in the presence of CXCL12 stimulation, while ELMO2 knockdown had no significant impact on the level of plasma membrane Gai2 (Figure 4C,D). Thus, Gai2 might be a key factor for chemokine-induced ELMO2 recruitment to the plasma membrane.

\section{Discussion}

Pancreatic cancer is one of the most malignant cancers, causing high morbidity and mortality. This is due to the intrinsic characteristics of this malignancy, such as rapid tissue invasion and metastasis. To acquire invasive and metastatic capabilities, cancer cells need to undergo multiple cellular changes, including oncogene-triggered signaling cascades. Several proteins are involved in these changes at the genetic and biochemical levels. ELMO family proteins are orthologs of $C$. elegans CED-12. They possess no catalytic activity, but associate with other proteins, serving as upstream activators and regulators of cytoskeletal rearrangements, thus favoring cell motility. Several studies have suggested a role of ELMO proteins in cancer. For instance, ELMO1 was clearly related to the invasive phenotype of glioma cells. In addition, the migratory and invasive abilities of glioma cells increase with the level of ELMO1 expression. Other studies have demonstrated that the overexpression of ELMO1 promotes cell motility and invasion in hepatocellular carcinoma and serous ovarian cancer ( $\mathrm{Li}$ et al. 2019; Wang et al. 2014). ELMO3 has also been reported to participate in events related to metastasis in several types of cancer, including lung cancer, colorectal cancer, and squamous-cell carcinoma of the head and neck (Fan et al. 2015; Kadletz et al. 2017; Peng et al. 2016). However, the function of ELMO2 in pancreatic cancer progression and metastasis has been poorly investigated. During the chemotaxis assay, a permeable filter separated the upper and lower wells of the Neuro Probe AP48 chemotaxis chamber. Interestingly, cell migration through the permeable filter could be inhibited when ELMO2 was reduced. Based on our data, the decrease in the number of migrated cells under ELMO2 knockdown condition without CXCL12 stimulation was statistically significant. The possible reason for this was that the decreased expression of ELMO2 reduced the migration capacity of tumor cells. In this study, we showed that ELMO2 knockdown inhibits CXCL12-mediated migration, chemotaxis, adhesion, and invasion of pancreatic cancer cells. CXCL12 interaction with its receptor, CXCR4, causes intracellular actin polymerization, which is necessary for pancreatic cancer cell migration and invasion. We found that stimulation with CXCL12 induces a noticeable increase in F-actin in pancreatic cancer cells, which can be prevented by ELMO2 knockdown. Therefore, ELMO2 is a boosting factor for the migration and metastasis of pancreatic cancer cells. Further studies will be needed to exhaustively characterize pathologically relevant ELMO2 interactions with other proteins. 
322 During the past few years, extensive efforts have been made to identify potential interactors of 323 ELMO proteins. It has been reported that brain-specific angiogenesis inhibitor (BAI3), a G 324 protein-coupled receptor binding to ELMO1, regulated myoblast fusion (Hamoud et al. 2014).

325 Moreover, a member of the Nck protein family, Nck-1, interacts with ELMO1 and controls the 326 activity of the Rho family GTPase, Rac1, which is involved in the reorganization of the actin 327 cytoskeleton. Furthermore, the membrane-bound proteins Gai2 and G $\beta \gamma$ have been found to 328 associate with ELMO1. These proteins activate downstream signaling factors that have an 329 impact on the restructuring of the actin cytoskeleton and promote the migration of cancer cells. 330 However, the mechanism by which ELMO2 interaction with its putative partner, Gai2, affects immunoprecipitation experiments demonstrated that exogenous ELMO2 directly interacted with endogenous Gai2, and vice versa. Interestingly, we found that the expression of GNAI2 had increased in the GV362-ELMO2 condition and when we overexpressed GNAI2 it seemed that also the expression level of ELMO2 increased. Overexpression of ELMO2 or Gai2 might force tumor cells to generate more endogenous Gai2 or ELMO2 to interact with in order to work together for directing migration and invasion of cancer. Future studies are needed to understand how these two proteins influence over the expression of one another. The interaction of ELMO2 with Gai2 was enhanced by CXCL12 stimulation. Moreover, CXCL12 stimulation promoted Gai2-mediated membrane translocation of ELMO2. Interestingly, when the expression of Gai2 was suppressed in human pancreatic cancer cell line PANC-1, ELMO2 translocation was substantially reduced, even in the presence of CXCL12 stimulation. It was suggested that Gai2 plays an indispensable role in ELMO2 translocation to the plasma membrane. Our results confirmed this finding and demonstrated that a physiologically relevant interaction between ELMO2 and Gai2 promoted actin polymerization in pancreatic cancer cells. We thus hypothesize that CXCL12 binding to the G-protein-coupled receptor, CXCR4, triggered a signal that was transmitted to the cell interior, causing ELMO2 recruitment to the plasma membrane. The latter event was dependent on Gai2. Thus, intracellular signals generated by the newly assembled CXCL12/ CXCR4 protein complex resulted in actin polymerization and invasive cell migration.

In summary, we showed that ELMO2 plays an essential role in CXCL12-mediated chemotaxis, migration, and invasion of human pancreatic cancer lines. Gai2 interacted directly with ELMO2 to promote metastatic changes. Considering these results, ELMO2 may be regarded as a promising target for the treatment of pancreatic cancer metastasis. Further research is needed to uncover the role of ELMO-related signaling in different types of cancer, identify valuable prognostic biomarkers, and develop therapeutic strategies centered on ELMO signaling.

\section{Acknowledgments}

We are much obliged to Professor Rong Wang (Central Laboratory, Xuan Wu Hospital, Capital Medical University), who kindly offered an experimental platform for our scientific research and provided proofreading assistance for this article. 
362

363

364

365

366

367

368

369

370

371

372

373

374

375

376

377

378

379

380

381

382

383

384

385

386

387

388

389

390

391

392

393

394

395

396

397

398

399

400

401

402

\section{Funding}

This work was supported by Beijing Hospitals Authority Youth Programme [grant number QMS20180805]; Cultivate Foundation of Capital Medical University [grant number PYZ2018154]; Top-notch Youth Project of the Supporting Plan for the Construction of Highlevel Teachers in Beijing-a $\square$ liated Universities [grant number CIT\&TCD201904093]; Beijing Municipal Commission of Science and Technology [grant number Z171100001017077]; Beijing Municipal Administration of Hospitals Clinical Medicine Development of Special Funding Support [grant number XMLX201404].

\section{Declaration of Interest: None}

\section{References}

Archibald KM, Kulbe H, Kwong J, Chakravarty P, Temple J, Chaplin T, Flak MB, McNeish IA, Deen S, Brenton JD, Young BD, and Balkwill F. 2012. Sequential genetic change at the TP53 and chemokine receptor CXCR4 locus during transformation of human ovarian surface epithelium. Oncogene 31:4987-4995. 10.1038/onc.2011.653

Balkwill F. 2004. Cancer and the chemokine network. Nat Rev Cancer 4:540-550. 10.1038/nrc1388

Condeelis J, and Segall JE. 2003. Intravital imaging of cell movement in tumours. Nat Rev Cancer 3:921-930. $10.1038 / \mathrm{nrc} 1231$

Condeelis J, Singer RH, and Segall JE. 2005. The great escape: when cancer cells hijack the genes for chemotaxis and motility. Annu Rev Cell Dev Biol 21:695-718. 10.1146/annurev.cellbio.21.122303.120306

Conroy T, Bachet JB, Ayav A, Huguet F, Lambert A, Caramella C, Marechal R, Van Laethem JL, and Ducreux M. 2016. Current standards and new innovative approaches for treatment of pancreatic cancer. Eur J Cancer 57:1022. 10.1016/j.ejca.2015.12.026

Fan W, Yang H, Xue H, Sun Y, and Zhang J. 2015. ELMO3 is a novel biomarker for diagnosis and prognosis of nonsmall cell lung cancer. Int J Clin Exp Pathol 8:5503-5508.

Fraser CC. 2008. G protein-coupled receptor connectivity to NF-kappaB in inflammation and cancer. Int Rev Immunol 27:320-350. 10.1080/08830180802262765

Fritsch R, de Krijger I, Fritsch K, George R, Reason B, Kumar MS, Diefenbacher M, Stamp G, and Downward J. 2013. RAS and RHO families of GTPases directly regulate distinct phosphoinositide 3-kinase isoforms. Cell 153:1050-1063. 10.1016/j.cell.2013.04.031

Garrido-Laguna I, and Hidalgo M. 2015. Pancreatic cancer: from state-of-the-art treatments to promising novel therapies. Nat Rev Clin Oncol 12:319-334. 10.1038/nrclinonc.2015.53

Gumienny TL, Brugnera E, Tosello-Trampont AC, Kinchen JM, Haney LB, Nishiwaki K, Walk SF, Nemergut ME, Macara IG, Francis R, SchedI T, Qin Y, Van Aelst L, Hengartner MO, and Ravichandran KS. 2001. CED12/ELMO, a novel member of the CrkII/Dock180/Rac pathway, is required for phagocytosis and cell migration. Cell 107:27-41. 10.1016/s0092-8674(01)00520-7

Guyon A. 2014. CXCL12 chemokine and its receptors as major players in the interactions between immune and nervous systems. Front Cell Neurosci 8:65. 10.3389/fncel.2014.00065

Hamoud N, Tran V, Croteau LP, Kania A, and Cote JF. 2014. G-protein coupled receptor BAI3 promotes myoblast 
403

404

405

406

407

408

409

410

411

412

413

414

415

416

417

418

419

420

421

422

423

424

425

426

427

428

429

430

431

432

433

434

435

436

437

438

439

440

441

442

443

fusion in vertebrates. Proc Natl Acad Sci U S A 111:3745-3750. 10.1073/pnas.1313886111

Hu TH, Yao Y, Yu S, Han LL, Wang WJ, Guo H, Tian T, Ruan ZP, Kang XM, Wang J, Wang SH, and Nan KJ. 2014. SDF1/CXCR4 promotes epithelial-mesenchymal transition and progression of colorectal cancer by activation of the Wnt/beta-catenin signaling pathway. Cancer Lett 354:417-426. 10.1016/j.canlet.2014.08.012

Hurst JH, and Hooks SB. 2009. Regulator of G-protein signaling (RGS) proteins in cancer biology. Biochem Pharmacol 78:1289-1297. 10.1016/j.bcp.2009.06.028

Iglesias PA, and Devreotes PN. 2008. Navigating through models of chemotaxis. Curr Opin Cell Biol 20:35-40. 10.1016/j.ceb.2007.11.011

Jarzynka MJ, Hu B, Hui KM, Bar-Joseph I, Gu W, Hirose T, Haney LB, Ravichandran KS, Nishikawa R, and Cheng SY. 2007. ELMO1 and Dock180, a bipartite Rac1 guanine nucleotide exchange factor, promote human glioma cell invasion. Cancer Res 67:7203-7211. 10.1158/0008-5472.CAN-07-0473

Jiang J, Liu G, Miao X, Hua S, and Zhong D. 2011. Overexpression of engulfment and cell motility 1 promotes cell invasion and migration of hepatocellular carcinoma. Exp Ther Med 2:505-511. 10.3892/etm.2011.229

Jin T, Xu X, Fang J, Isik N, Yan J, Brzostowski JA, and Hereld D. 2009. How human leukocytes track down and destroy pathogens: lessons learned from the model organism Dictyostelium discoideum. Immunol Res 43:118-127. 10.1007/s12026-008-8056-7

Kadletz L, Heiduschka G, Wiebringhaus R, Gurnhofer E, Kotowski U, Haymerle G, Brunner M, Barry C, and Kenner L. 2017. ELMO3 expression indicates a poor prognosis in head and neck squamous cell carcinoma - a short report. Cell Oncol (Dordr) 40:193-198. 10.1007/s13402-016-0310-8

Kim M, Kim M, Lee S, Kuninaka S, Saya H, Lee H, Lee S, and Lim DS. 2013. cAMP/PKA signalling reinforces the LATSYAP pathway to fully suppress YAP in response to actin cytoskeletal changes. EMBO J 32:1543-1555. 10.1038/emboj.2013.102

Kleeff J, Korc M, Apte M, La Vecchia C, Johnson CD, Biankin AV, Neale RE, Tempero M, Tuveson DA, Hruban RH, and Neoptolemos JP. 2016. Pancreatic cancer. Nat Rev Dis Primers 2:16022. 10.1038/nrdp.2016.22

Li H, Wang Y, Lu Y, and Li F. 2019. Annexin A2 interacting with ELMO1 regulates HCC chemotaxis and metastasis. Life Sci 222:168-174. 10.1016/j.Ifs.2019.03.003

Li H, Yang L, Fu H, Yan J, Wang Y, Guo H, Hao X, Xu X, Jin T, and Zhang N. 2013. Association between Galphai2 and ELMO1/Dock180 connects chemokine signalling with Rac activation and metastasis. Nat Commun 4:1706. $10.1038 /$ ncomms 2680

Li X, Li P, Chang Y, Xu Q, Wu Z, Ma Q, and Wang Z. 2014. The SDF-1/CXCR4 axis induces epithelial-mesenchymal transition in hepatocellular carcinoma. Mol Cell Biochem 392:77-84. 10.1007/s11010-014-2020-8

Muller A, Homey B, Soto H, Ge N, Catron D, Buchanan ME, McClanahan T, Murphy E, Yuan W, Wagner SN, Barrera $\mathrm{JL}$, Mohar A, Verastegui E, and Zlotnik A. 2001. Involvement of chemokine receptors in breast cancer metastasis. Nature 410:50-56. 10.1038/35065016

Murphy PM. 2001. Chemokines and the molecular basis of cancer metastasis. N Engl J Med 345:833-835. 10.1056/NEJM200109133451113

Patel M, Pelletier A, and Cote JF. 2011. Opening up on ELMO regulation: New insights into the control of Rac signaling by the DOCK180/ELMO complex. Small GTPases 2:268-275. 10.4161/sgtp.2.5.17716

Peng HY, Yu QF, Shen W, Guo CM, Li Z, Zhou XY, Zhou NJ, Min WP, and Gao D. 2016. Knockdown of ELMO3 Suppresses Growth, Invasion and Metastasis of Colorectal Cancer. Int J Mol Sci 17. 10.3390/ijms17122119

Siegel RL, Miller KD, and Jemal A. 2018. Cancer statistics, 2018. CA Cancer J Clin 68:7-30. 10.3322/caac.21442

Peer) reviewing PDF | (2019:12:44440:2:0:NEW 10 Mar 2020) 
Sun R, Gao P, Chen L, Ma D, Wang J, Oppenheim JJ, and Zhang N. 2005. Protein kinase C zeta is required for epidermal growth factor-induced chemotaxis of human breast cancer cells. Cancer Res 65:1433-1441. 10.1158/0008-5472.CAN-04-1163

Swaney KF, Huang CH, and Devreotes PN. 2010. Eukaryotic chemotaxis: a network of signaling pathways controls motility, directional sensing, and polarity. Annu Rev Biophys 39:265-289. 10.1146/annurev.biophys.093008.131228

Toret CP, Collins C, and Nelson WJ. 2014. An Elmo-Dock complex locally controls Rho GTPases and actin remodeling during cadherin-mediated adhesion. J Cell Biol 207:577-587. 10.1083/jcb.201406135

Tsuboi S. 2006. A complex of Wiskott-Aldrich syndrome protein with mammalian verprolins plays an important role in monocyte chemotaxis. J Immunol 176:6576-6585. 10.4049/jimmunol.176.11.6576

Wang J, Dai JM, Che YL, Gao YM, Peng HJ, Liu B, Wang H, and Linghu H. 2014. Elmo1 helps dock180 to regulate Rac1 activity and cell migration of ovarian cancer. Int J Gynecol Cancer 24:844-850. 10.1097/IGC.0000000000000137

Xu X, Meckel T, Brzostowski JA, Yan J, Meier-Schellersheim M, and Jin T. 2010. Coupling mechanism of a GPCR and a heterotrimeric $\mathrm{G}$ protein during chemoattractant gradient sensing in Dictyostelium. Sci Signal 3:ra71. 10.1126/scisignal.2000980

Yan J, Mihaylov V, Xu X, Brzostowski JA, Li H, Liu L, Veenstra TD, Parent CA, and Jin T. 2012. A Gbetagamma effector, ElmoE, transduces GPCR signaling to the actin network during chemotaxis. Dev Cell 22:92-103. 10.1016/j.devcel.2011.11.007

Yang P, Wang G, Huo H, Li Q, Zhao Y, and Liu Y. 2015. SDF-1/CXCR4 signaling up-regulates survivin to regulate human sacral chondrosarcoma cell cycle and epithelial-mesenchymal transition via ERK and PI3K/AKT pathway. Med Oncol 32:377. 10.1007/s12032-014-0377-x

Zhang B, Shi L, Lu S, Sun X, Liu Y, Li H, Wang X, Zhao C, Zhang H, and Wang Y. 2015. Autocrine IL-8 promotes F-actin polymerization and mediate mesenchymal transition via ELMO1-NF-kappaB-Snail signaling in glioma. Cancer Biol Ther 16:898-911. 10.1080/15384047.2015.1028702

Zhang G, Chen X, Qiu F, Zhu F, Lei W, and Nie J. 2014. A novel interaction between the SH2 domain of signaling adaptor protein Nck-1 and the upstream regulator of the Rho family GTPase Rac1 engulfment and cell motility 1 (ELMO1) promotes Rac1 activation and cell motility. J Biol Chem 289:23112-23122. 10.1074/jbc.M114.549550

\section{Figure Legends}

Fig. 1. Function of ELMO2 in pancreatic cancer cell migration and chemotaxis. (A) Western blot shows an evident knockdown of ELMO2 in human pancreatic cell lines. GAPDH was used as a loading control for western blot. (B) Chemotaxis in ELMO2 knockdown cells (data are the mean of three independent experiments; two-way ANOVA, $* * p<0.001$ ). (C) Wound healing assay in siELMO2 cells. Pancreatic cancer cells were seeded in 6-well plates and incubated until the monolayer reached $80-90 \%$ confluence (ca. $24 \mathrm{~h}$ of growth). The medium was replaced by an RPMI 1640 medium containing $1 \%$ fetal bovine serum. The gap distance was measured at $0,3,6,9,12$, and $24 \mathrm{~h}$ (data are the mean of three independent experiments; twoway ANOVA, ${ }^{* *} \mathrm{p}<0.001$ ). 
485

486

487

488

489

490

491

492

493

494

495

496

497

498

499

500

501

502

503

504

505

506

507

508

509

510

511

512

513

514

515

516

Fig. 2. Knockdown of ELMO2 inhibited F-actin polymerization and invasion in pancreatic cancer cells. (A) The invasion assay showed that ELMO2 knockdown decreased the CXCL12mediated invasive abilities of PANC-1 and AsPC-1 cells (data are the mean of three independent experiments; two-way ANOVA, ${ }^{* *} p<0.001$ ). (B) Adhesion assay in PANC-1 and AsPC-1 cells. The cell adhesion rate was much higher in normal cells than in siELMO2 cells (data are the mean of three independent experiments; two-way ANOVA, **p < 0.001). (C) ELMO2 knockdown reduced actin polymerization in pancreatic cancer cells. F-actin value was measured at different time points $(0,4,8,15,30,60,120$, and $300 \mathrm{~s})$. Time course of relative F-actin content in normal, control, and siELMO2 cells upon CXCL12 stimulation (data are the mean of three independent experiments; two-way ANOVA, $* * p<0.001)$.

Fig. 3. ELMO2 interacts with Gai2. (A) PANC-1 cells were transfected with GV362 FlagELMO2 to increase ELMO2 expression. Cell lysates were immunoprecipitated with anti-Flag antibody, and magnetic beads were used to capture the immune complexes. The eluted proteins were separated by SDS-PAGE and detected with specific antibodies. (B) Exogenous Gai2 was overexpressed by transfection with the GV141-GNAI2-Flag plasmid. Proteins of the immune complex were pulled down by anti-Flag antibody and detected by anti-ELMO2 or anti-Gai2 antibodies. Input was the total protein lysates which were prepared from cells with RIPA lysis buffer. IP referred to the immunoprecipitate that was eluted from the beads after the immunoprecipitation.

Fig. 4. CXCL12 stimulation results in ELMO2 membrane translocation. (A) Plasma membrane colocalizations of Gai2 and ELMO2 were evident upon pancreatic cancer cell stimulation with CXCL12. The extent of colocalization was calculated through ImageJ software. (B) No significant changes in plasma membrane-associated Gai2 fluorescence were detected in ELMO2 knockdown cells, even with CXCL12 stimulation. One-way ANOVA, p > 0.05. (C) Western blot clearly shows the Gai2 knockdown in siRNA-transfected PANC-1 cells. GAPDH was used as a loading control. (D) ELMO2 membrane translocation was reduced in Gai2 knockdown cells, even in the presence of CXCL12 stimulation. Twenty-five images were analyzed by ImageJ software. One-way ANOVA, $* * p<0.001$. The arrows indicated the plasma membrane colocalization of Gai2 or ELMO2. 
Figure 1

Function of ELMO2 in pancreatic cancer cell migration and chemotaxis.

(A) Western blot shows an evident knockdown of ELMO2 in human pancreatic cell lines.

GAPDH was used as a loading control for western blot. (B) Chemotaxis in ELMO2 knockdown cells (data are the mean of three independent experiments; two-way ANOVA, ${ }^{* *} p<0.001$ ).

(C) Wound healing assay in siELMO2 cells. Pancreatic cancer cells were seeded in 6-well plates and incubated until the monolayer reached $80-90 \%$ confluence (ca. $24 \mathrm{~h}$ of growth). The medium was replaced by an RPMI 1640 medium containing 1\% fetal bovine serum. The gap distance was measured at $0,3,6,9,12$, and $24 \mathrm{~h}$ (data are the mean of three independent experiments; two-way ANOVA, ${ }^{* *} p<0.001$ ).

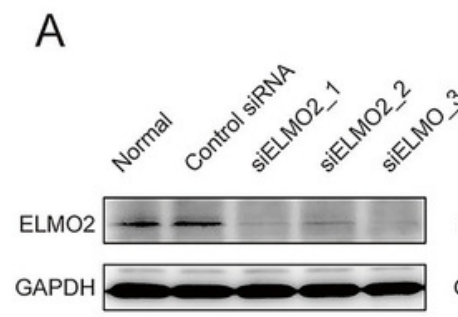

PANC-1

C

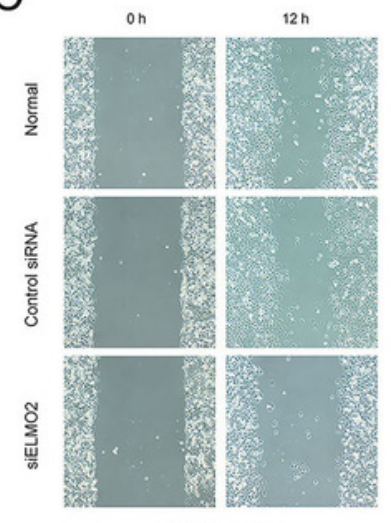

PANC-1

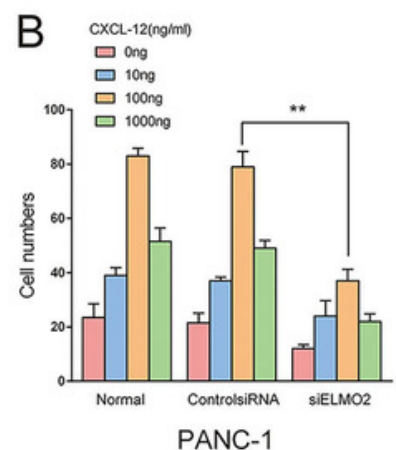

PANC-1

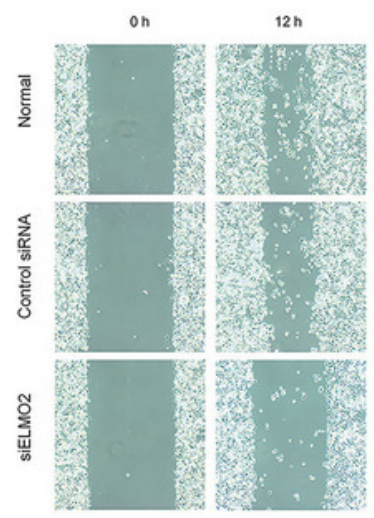

AsPC-1
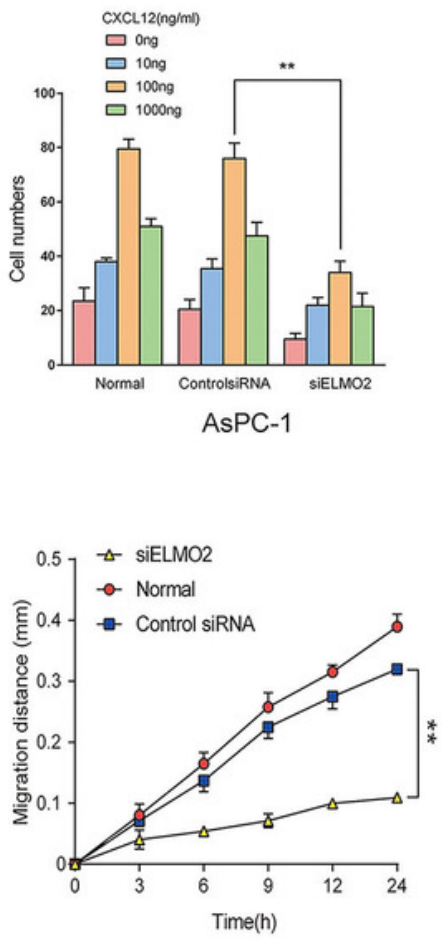


\section{Figure 2}

Knockdown of ELMO2 inhibited F-actin polymerization and invasion in pancreatic cancer cells.

(A) The invasion assay showed that ELMO2 knockdown decreased the CXCL12-mediated invasive abilities of PANC-1 and AsPC-1 cells (data are the mean of three independent experiments; two-way ANOVA, ${ }^{* *} p<0.001$ ). (B) Adhesion assay in PANC-1 and AsPC-1 cells. The cell adhesion rate was much higher in normal cells than in siELMO2 cells (data are the mean of three independent experiments; two-way ANOVA, $* * p<0.001$ ). (C) ELMO2 knockdown reduced actin polymerization in pancreatic cancer cells. F-actin value was measured at different time points $(0,4,8,15,30,60,120$, and 300 s). Time course of relative F-actin content in normal, control, and siELMO2 cells upon CXCL12 stimulation (data are the mean of three independent experiments; two-way ANOVA, ${ }^{* *} p<0.001$ ). 
A
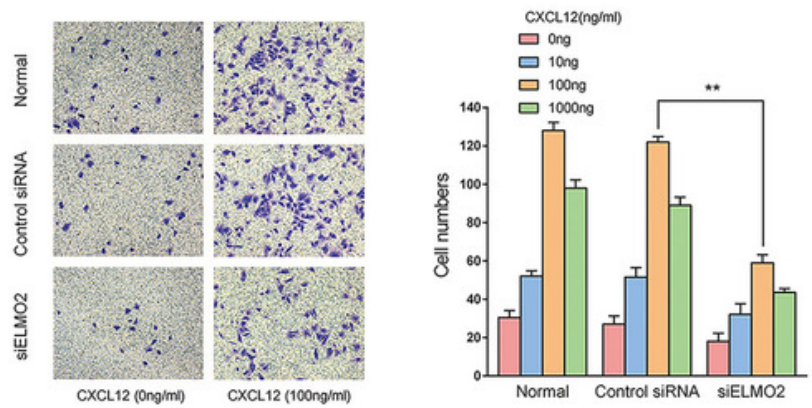

PANC-1
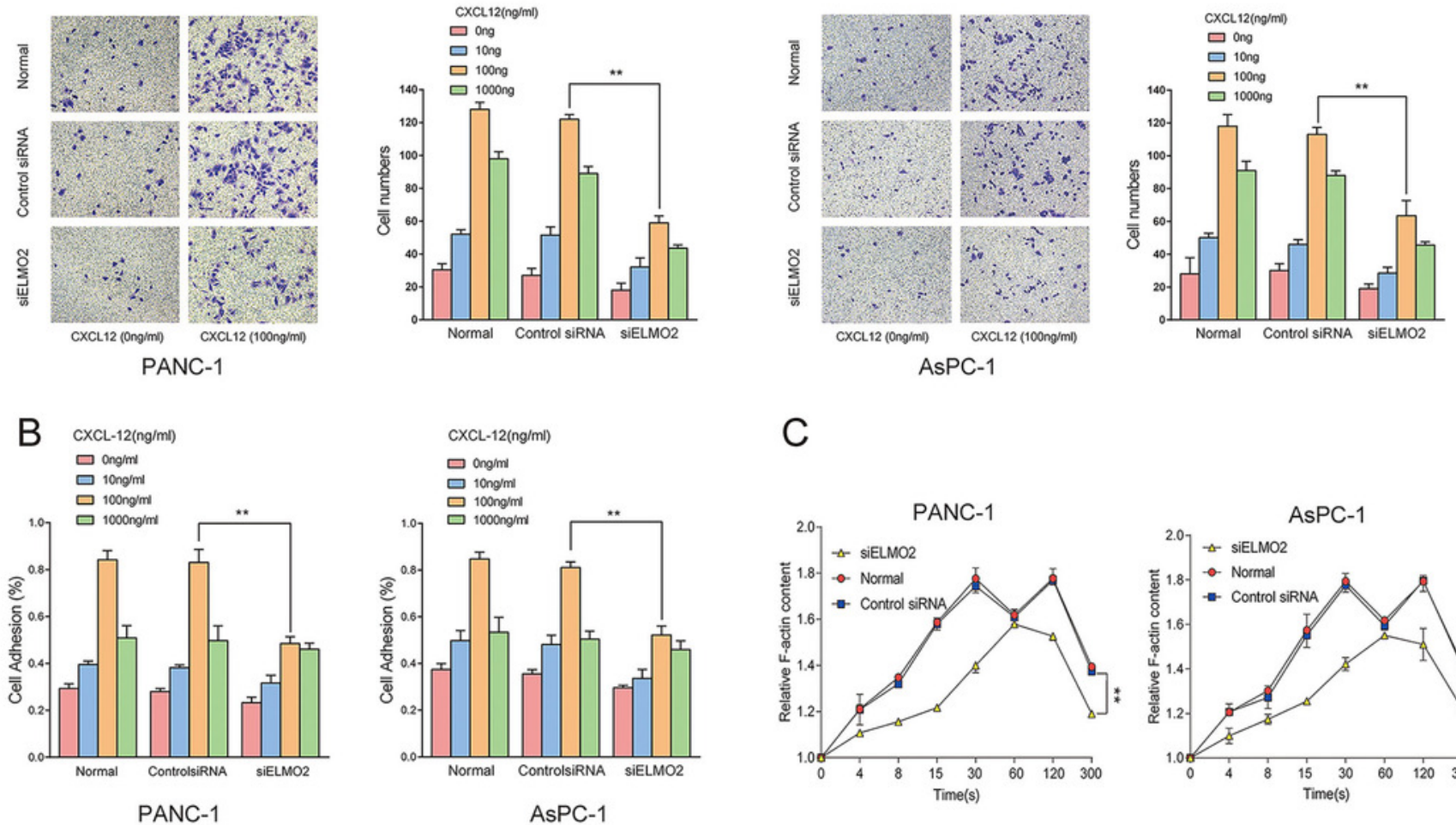

C
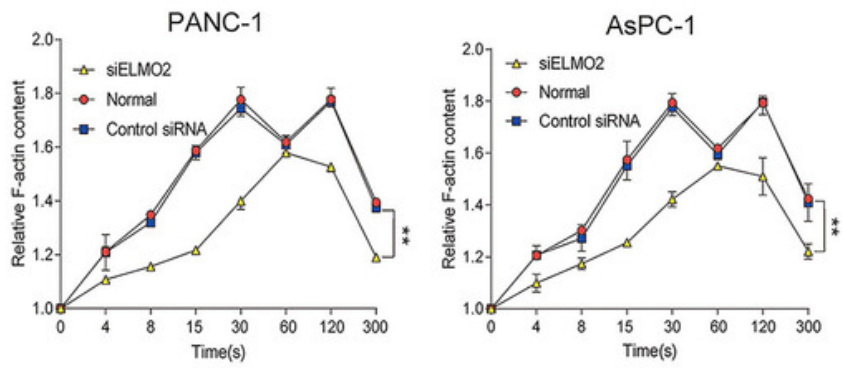


\section{Figure 3}

ELMO2 interacts with Gai2.

(A) PANC-1 cells were transfected with GV362 Flag-ELMO2 to increase ELMO2 expression.

Cell lysates were immunoprecipitated with anti-Flag antibody, and magnetic beads were used to capture the immune complexes. The eluted proteins were separated by SDS-PAGE and detected with specific antibodies. (B) Exogenous Gai2 was overexpressed by transfection with the GV141-GNAI2-Flag plasmid. Proteins of the immune complex were pulled down by anti-Flag antibody and detected by anti-ELMO2 or anti-Gai2 antibodies. Input was the total protein lysates which were prepared from cells with RIPA lysis buffer. IP referred to the immunoprecipitate that was eluted from the beads after the immunoprecipitation.
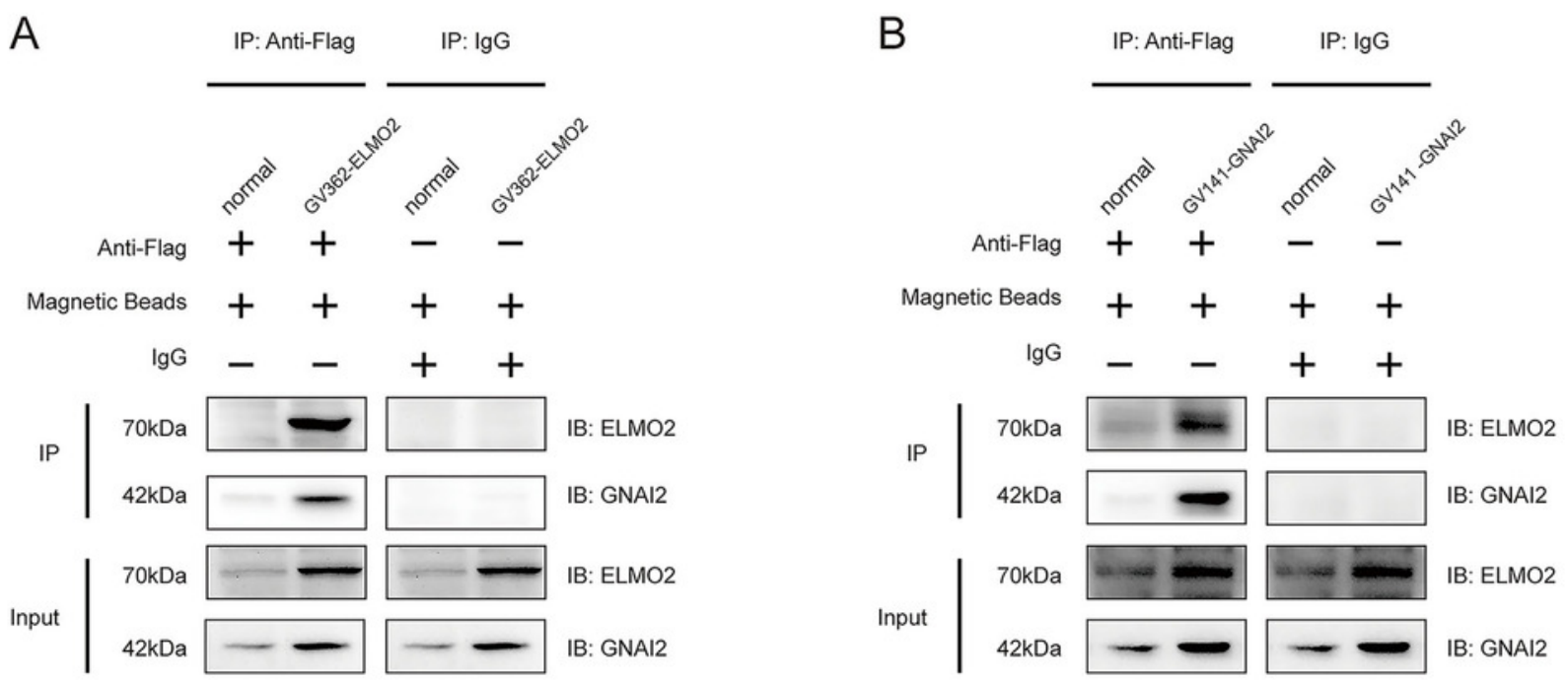


\section{Figure 4}

CXCL12 stimulation results in ELMO2 membrane translocation.

(A) Plasma membrane colocalizations of Gai2 and ELMO2 were evident upon pancreatic cancer cell stimulation with CXCL12. The extent of colocalization was calculated through ImageJ software. (B) No significant changes in plasma membrane-associated Gai2 fluorescence were detected in ELMO2 knockdown cells, even with CXCL12 stimulation. Oneway ANOVA, $p>0.05$. (C) Western blot clearly shows the Gai2 knockdown in siRNAtransfected PANC-1 cells. GAPDH was used as a loading control. (D) ELMO2 membrane translocation was reduced in Gai2 knockdown cells, even in the presence of CXCL12 stimulation. Twenty-five images were analyzed by ImageJ software. One-way ANOVA, **p < 0.001 . The arrows indicated the plasma membrane colocalization of Gai2 or ELMO2. 

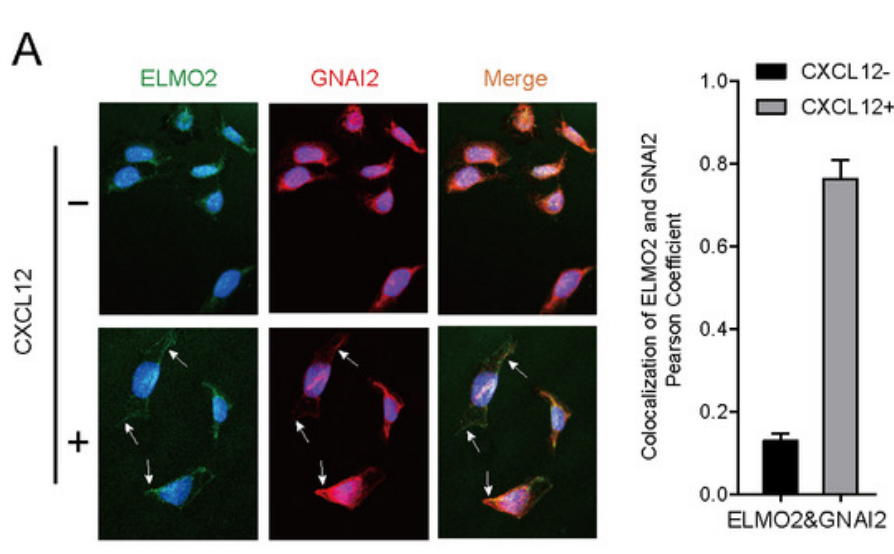

C

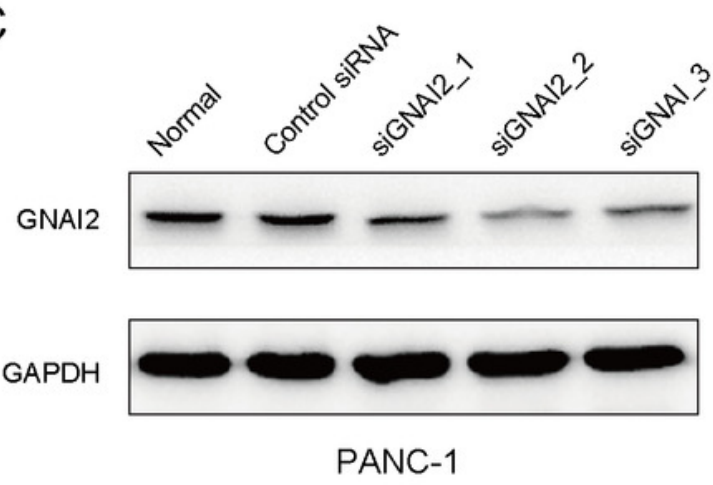

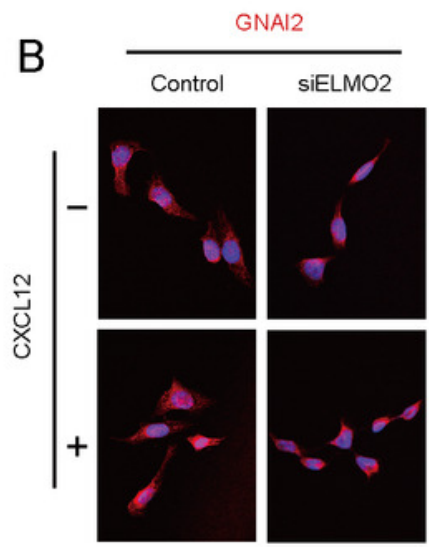
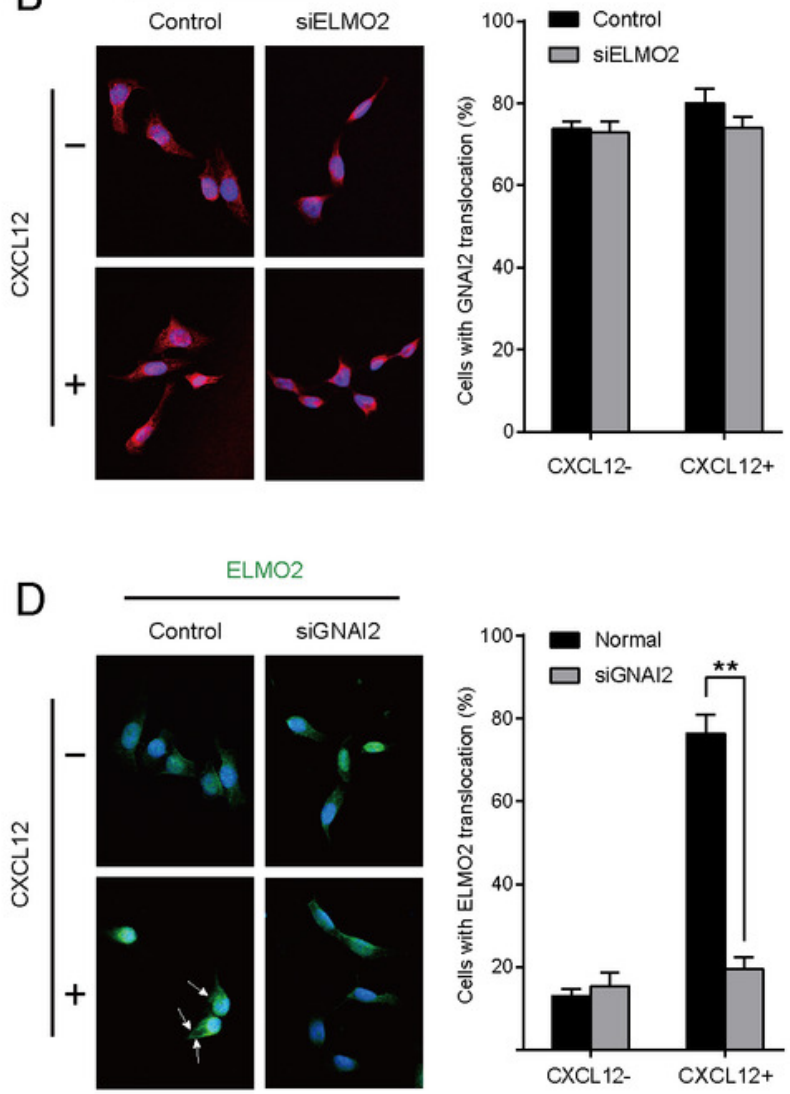